\title{
Remarkable Predictions of Classical Electrodynamics on Elementary Charge and the Energy Density of Vacuum
}

\author{
Vernon Cooray ${ }^{1}$, Gerald Cooray ${ }^{2}$ \\ ${ }^{1}$ Department of Engineering Sciences, Uppsala University, Uppsala, Sweden \\ ${ }^{2}$ Karolinska Institute, Stockholm, Sweden \\ Email: Vernon.Cooray@angstrom.uu.se
}

How to cite this paper: Cooray, V. and Cooray, G. (2018) Remarkable Predictions of Classical Electrodynamics on Elementary Charge and the Energy Density of Vacuum. Journal of Electromagnetic Analysis and Applications, 10, 77-87.

https://doi.org/10.4236/jemaa.2018.105006

Received: March 21, 2018

Accepted: May 20, 2018

Published: May 23, 2018

Copyright ( $) 2018$ by authors and Scientific Research Publishing Inc. This work is licensed under the Creative Commons Attribution International License (CC BY 4.0).

http://creativecommons.org/licenses/by/4.0/

\begin{abstract}
In a recent paper, we have studied the nature of the electromagnetic energy radiated over a single period of oscillation by an antenna working in frequency domain under ideal conditions and without losses when the oscillating charge in the antenna is reduced to the elementary charge. Here we extend and expand that study. The energy radiated by an oscillating current in an antenna occurs in bursts of duration $T / 2$, where $T$ is the period of oscillation. The results obtained here, based purely on classical electrodynamics, can be summarized by the inequality $U \geq h v \rightarrow q_{0} \geq e$ where $U$ is the energy radiated in a single burst of duration $T / 2, h$ is the Planck constant, $v$ is the frequency of oscillation and $q_{0}$ is the magnitude of the oscillating charge associated with the current. The condition $U=h v \rightarrow q_{0}=e$ is obtained when the length of the antenna is equal to the ultimate Hubble radius of the universe (i.e. the maximum value of the antenna length allowed by nature) and the wavelength is equal to the Bohr radius (resulting from the smallest possible radius of the conductor allowed by nature). The ultimate Hubble radius is directly related to the vacuum energy density. The inequality obtained here is in general agreement with the one obtained in the previous study. One novel feature of this extended analysis is the discovery of an expression, in terms of the elementary charge and other atomic constants, for the vacuum energy density of the universe. This expression predicts the vacuum energy density to be about $4 \times 10^{-10} \mathrm{~J} / \mathrm{m}^{3}$ which is in reasonable agreement with the measured value of $6 \times 10^{-10} \mathrm{~J} / \mathrm{m}^{3}$.
\end{abstract}

\section{Keywords}

Classical Electrodynamics, Electromagnetic Radiation, Oscillating Currents, 
Energy of a Photon, Radius of the Universe, Hubble Radius, Vacuum Energy, Dark Energy, Elementary Charge, Electronic Charge

\section{Introduction}

In several recent publications, Cooray and Cooray [1] [2] investigated the lower limits of the radiated energy when the charge associated with the current in the antenna giving rise to the radiation is equal to the elementary charge. Based on the work done, it was shown that the time domain radiation fields satisfy the order of magnitude relationship, $A \geq h / 4 \pi \rightarrow q \geq e$, where $A$ is the action associated with the energy radiated (i.e. product of energy and the time of emission), $q$ is the charge associated with the impulse current in the transmitting antenna, and $h$ is the Planck constant. The same relationship is shown to be valid for the energy radiated when a relativistic charged particle is suddenly stopped by a perfectly conducting plane (i.e. transition radiation). In the case of antennas working in frequency domain it was shown in reference [1] (hereinafter referred to as Paper 1), that the radiation satisfy the order of magnitude relationship, $U \geq h v \rightarrow q \geq e$ where $U$ is the energy dissipated over a period of oscillation and $q$ is the charge associated with the oscillating current [1]. The results obtained in Paper 1 can be improved in several aspects. First, in that paper the energy dissipated over a given period of time was expressed as an integral and it was evaluated using numerical techniques. Further investigations by us show that, due to the rapidly oscillating nature of the integrand, the rounding off errors associated with the numerical integration could have clamped the energy to a constant value at extremely high values of $L / \lambda$, where $L$ is the antenna length and $\lambda$ is the wavelength. Subsequent analysis showed that the energy increases slowly (i.e. logarithmically) instead of reaching a steady value. This slowly increasing nature of the emitted energy over a given time period with increasing $L / \lambda$ and the limited range of $L / \lambda$ over which the analysis was carried out in Paper 1 compelled us to revisit the work.

\section{Analysis}

The starting point of the analysis is a monopole antenna of length $L / 2$ working in frequency domain located in free space over a perfectly conducting ground plane. The antenna is located along the $\mathrm{z}$-axis and the origin of the Cartesian coordinate system to be used in the analysis is located at the point where the antenna meets the perfectly conducting ground plane. The geometry of the problem is shown in Figure 1. Together with its image in the perfectly conducting ground plane, the antenna functions as a dipole of length $L$ radiating into the upper half space (region above the perfectly conducting plane). The radius of the antenna is denoted by $a$. The antenna is fed by a current source located at the ground end. 


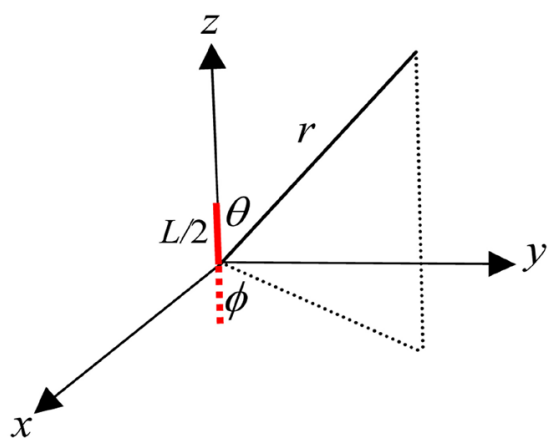

Figure 1. The geometry relevant to the description of electromagnetic fields of an antenna of length $L / 2$ located over a perfectly conducting ground plane. The perfectly conducting ground plane coincides with the $x-y$ plane. The antenna is directed along the $\mathrm{z}$-axis. The dotted line indicates the image of the antenna in the ground plane.

The one and the only assumption that is being made in Paper 1, and also here, is the existence of ideal conditions where all the losses associated with thermal, dispersion and radiation as the current oscillates along the antenna can be neglected. This indeed is far from reality and we will discuss the effects of these losses on the results in the discussion section. Under this assumed ideal conditions the current distribution along the antenna including its image in the perfectly conducting ground plane is given by [3] [4]

$$
\begin{gathered}
I(t, z)=I_{0} \sin \left\{\frac{2 \pi}{\lambda}\left(\frac{L}{2}-z\right)\right\} \mathrm{e}^{j \omega t}, \quad 0 \leq z \leq L / 2 \\
I(t, z)=I_{0} \sin \left\{\frac{2 \pi}{\lambda}\left(\frac{L}{2}+z\right)\right\} \mathrm{e}^{j \omega t}, \quad-L / 2 \leq z \leq 0
\end{gathered}
$$

With this current distribution, the total power dissipation, $P_{T}$ is given by [4]

$$
P_{T}=\frac{\sin ^{2}(2 \pi t / T) I_{0}^{2}}{2 \pi \varepsilon_{0} c} \int_{0}^{\pi / 2}\left[\cos \left(\frac{k L}{2} \cos \theta\right)-\cos \left(\frac{k L}{2}\right)\right]^{2} \mathrm{~d} \theta
$$

In the above equation $k=2 \pi / \lambda$ where $\lambda$ is the wavelength, $t$ is the time and $T$ is the period of oscillation. This equation is valid when $\lambda \gg a$, where $a$ is the radius of the conductor [4]. The angle $\theta$ is defined in Figure 1 . The average total power, $P_{a v}$, is then given by

$$
P_{a v}=\frac{I_{0}^{2}}{4 \pi \varepsilon_{0} c} \int_{0}^{\pi / 2}\left[\cos \left(\frac{k L}{2} \cos \theta\right)-\cos \left(\frac{k L}{2}\right)\right]^{2} \mathrm{~d} \theta
$$

If the oscillating charge associated with the current is given by $q=q_{0} \mathrm{e}^{j 2 \pi v t}$ (note that $j^{2}=-1$ ), Equation (4) can be written as

$$
P_{a v}=\frac{q_{0}^{2}(2 \pi v)^{2}}{4 \pi \varepsilon_{0} c} \int_{0}^{\pi / 2}\left[\cos \left(\frac{k L}{2} \cos \theta\right)-\cos \left(\frac{k L}{2}\right)\right]^{2} \mathrm{~d} \theta
$$


If $L / \lambda \ll 1$ the integral in Equation (5) can easily be solved resulting in

$$
P_{a v}=\frac{q_{0}^{2}(2 \pi v)^{2}}{\varepsilon_{0} c} \frac{\pi}{6}\left(\frac{l}{\lambda}\right)^{2}
$$

In Paper 1, when $L / \lambda$ is comparable to or larger than unity, the integral in Equation (5) was solved numerically. As one can see, for very large values of $k L$ (or $L / \lambda$ ) the integrand in Equation (5) oscillates rapidly as a function of $\theta$. This could introduce significant errors in the numerical integration specially when the value of $(k L)^{-1}$ becomes comparable to the computational accuracy of the computer. Fortunately, an analytical expression in terms of Cosine and Sine Integrals is available for this integral [4]. Using that analytical expression for the integral, the average total power radiated by the antenna can be written as

$$
\begin{aligned}
P_{a v}= & \frac{q_{0}^{2}(2 \pi v)^{2}}{8 \pi \varepsilon_{0} c}\left\{\gamma+\ln (k L)-C_{i}(k L)+\frac{1}{2} \sin (k L)\left[S_{i}(2 k L)-2 S_{i}(k L)\right]\right. \\
& \left.+\frac{1}{2} \cos (k L)\left[\gamma+\ln (k L / 2)+C_{i}(2 k L)-2 C_{i}(k L)\right]\right\}
\end{aligned}
$$

In the above equation, $C_{i}$ is the Cosine Integral, $S_{i}$ is the Sine Integral and $\gamma$ is the Euler-Mascheroni constant.

\section{Results}

First of all observe that from Equation (3) the power generated by the antenna consists of bursts of energy each burst having a duration of $T / 2$, where $T$ is the period of oscillation. This is due to the fact that the radiated power is proportional to $\sin ^{2}(2 \pi t / T)$. For example, Figure 2 illustrates the variation of total power (with peak normalized to unity) as a function of $t / T$ for any given value of

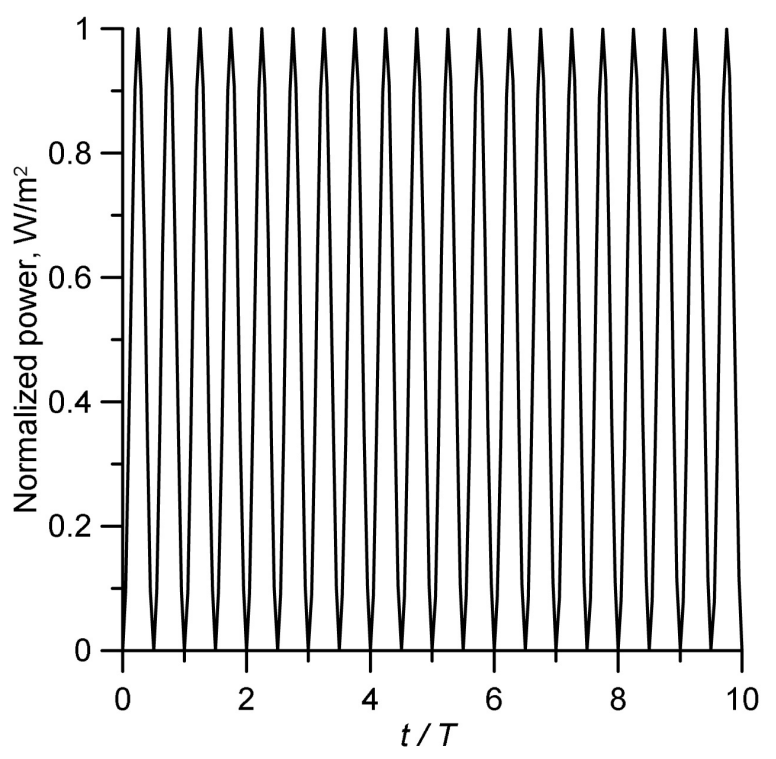

Figure 2. The normalized total power dissipation as given by Equation (3) for any given value of $k L$ as a function of $t / T$ where $t$ is the time and $T$ is the period. 
$k L$. This shows that the radiated energy can be separated into individual bursts of duration $T / 2$. Let us consider the radiated energy associated with a single burst of energy. This energy, $U$, is given by

$$
\begin{aligned}
U= & \frac{q_{0}^{2} \pi v^{2}}{2 \varepsilon_{0} c} \frac{T}{2}\left\{\gamma+\ln (k L)-C_{i}(k L)+\frac{1}{2} \sin (k L)\left[S_{i}(2 k L)-2 S_{i}(k L)\right]\right. \\
& \left.+\frac{1}{2} \cos (k L)\left[\gamma+\ln (k L / 2)+C_{i}(2 k L)-2 C_{i}(k L)\right]\right\}
\end{aligned}
$$

This can be written as

$$
\begin{aligned}
U= & \frac{q_{0}^{2} \pi \nu}{4 \varepsilon_{0} c}\left\{\gamma+\ln (k L)-C_{i}(k L)+\frac{1}{2} \sin (k L)\left[S_{i}(2 k L)-2 S_{i}(k L)\right]\right. \\
& \left.+\frac{1}{2} \cos (k L)\left[\gamma+\ln (k L / 2)+C_{i}(2 k L)-2 C_{i}(k L)\right]\right\}
\end{aligned}
$$

Observe from Equation (9) that for large values of $k L$ the value of $U$ oscillates rapidly with $k L$. The upper and lower bounds of $U$ occur when $k L=n \pi$ and $k L=m \pi$ where $n$ and $m$ are even and odd integers (i.e. when $\cos (k L)=1$ or $\cos (k L)=-1$ ). The median value of $U$ is given by

$$
U_{m}=\frac{q_{0}^{2} \pi v}{4 \varepsilon_{0} c}\left\{\gamma+\ln (k L)-C_{i}(k L)\right\}
$$

First, note that for large $k L$ the Cosine Integral varies as $\sim \cos (k L) /(k L)^{2}$ and it can be neglected with respect to other terms when $k L$ is very large. Thus for very large $k L$ the function $U_{m}$ reduces to the following

$$
U_{m}=\frac{q_{0}^{2} \pi v}{4 \varepsilon_{0} c}\{\gamma+\ln (k L)\}
$$

Observe that this function goes to infinity as $k L$ or $L / \lambda$ goes to infinity. However, in reality, $L / \lambda$ is limited by natural bounds. The magnitude of these natural bounds has been discussed in previous publications [5] [6]. First, the length of the antenna $L / 2$ cannot be larger than the Hubble radius of the universe. This is the maximum length that can be realized in the universe by any given observer. The Hubble radius continues to increase at the current cosmological time and the maximum length that can ever be achieved by an observer is the limiting value (or the upper limit) of the Hubble radius of the universe. Let us denote this limiting Hubble radius by the parameter $R_{\infty}$. Second, the radius of the antenna cannot be smaller than the Bohr radius $a_{0}$ and, therefore, the lower bound for the wavelength of oscillation is also equal to $a_{0}$. Thus the limiting value of the ratio $L / \lambda$ is equal to $2 R_{\infty} / a_{0}$ and any value large than this will not make any physical sense. Thus, the maximum value of $U_{m}$ that can ever be realized in nature for a given oscillating charge is given by

$$
U_{m m}=\frac{q_{0}^{2} \pi v}{4 \varepsilon_{0} c}\left\{\gamma+\ln \left(4 \pi R_{\infty} / a_{0}\right)\right\}
$$

Let us consider the number of energy units, equivalent to the energy of a photon, associated with this radiated energy. Since the energy of a single photon 
is equal to $h v$ the number of such energy units associated with this energy is given by

$$
N=\frac{q_{0}^{2} \pi}{4 \varepsilon_{0} h c}\left\{\gamma+\ln \left(4 \pi R_{\infty} / a_{0}\right)\right\}
$$

This equation shows that the number of such energy units decreases with decreasing charge. The charge associated with a given number of such energy units is given by

$$
q_{0}=\sqrt{\frac{4 \varepsilon_{0} h c N}{\pi\left\{\gamma+\ln \left(4 \pi R_{\infty} / a_{0}\right)\right\}}}
$$

When the number of such energy units (or the number of equivalent photons) is reduced to one, the oscillating charge reduces to

$$
q_{0}=\sqrt{\frac{4 \varepsilon_{0} h c}{\pi\left\{\gamma+\ln \left(4 \pi R_{\infty} / a_{0}\right)\right\}}}
$$

Interestingly, information concerning the maximum possible value of the Hubble radius is ingrained into the fabric of the universe through the magnitude of the vacuum energy density. If the density of the vacuum energy, $\rho_{\Lambda}$, of a flat universe is zero then the ultimate size of the Hubble radius is infinity [7]. However, if the vacuum energy density is finite, the Hubble radius will reach a finite value as the cosmological time increases. The experimental evidence shows that the vacuum density in the universe is not zero but has a small value of about $6 \times 10^{-10} \mathrm{~J} / \mathrm{m}^{3}$ [8]. With a finite vacuum energy density the maximum or the ultimate Hubble radius is given by [7] [9]

$$
R_{\infty}=c^{2} \sqrt{\frac{3}{8 \pi G \rho_{\Lambda}}}
$$

Since this is the maximum ever possible value of the Hubble radius, and its value is determined by the microscopic parameters of the fabric of space, it is the correct parameter to be inserted into Equation (15). With this value for the Hubble radius Equation (15) can be written as

$$
q_{0}=\sqrt{\pi\left\{\gamma+\ln \left[\frac{4 \pi c^{2}}{a_{0}} \sqrt{\left.\frac{3}{8 \pi G \rho_{\Lambda}}\right]}\right\}\right.}
$$

Substituting values for all the known parameters in Equation (17) we obtain $q_{0}=1.603 \times 10^{-19} \mathrm{C}$, which is within $0.1 \%$ of the elementary charge. It is important to point out here that, had we used the current Hubble radius corresponding to cosmological times spanning from less than 0.1 billion years until the time at which the Hubble radius had reached a steady value, the values of $q_{0}$ we would have obtained would still remain within the order of magnitude of the elementary charge. This shows that when the length of the antenna is stretched and the radius of the antenna is compressed to its natural limits, and oscillating charge in the antenna is equal to the elementary charge, the median value of the energy 
it radiates during each half period of oscillation is equal to that of a photon corresponding to the frequency of oscillation. The Bohr radius is given by $a_{0}=\varepsilon_{0} h^{2} / \pi m_{e} e^{2}$ and substituting this expression for the Bohr radius in Equation (17) we obtain

$$
q_{0}=\sqrt{\frac{4 \varepsilon_{0} h c}{\pi\left\{\gamma+\ln \left[\frac{4 \pi^{2} c^{2} m_{e} e^{2}}{\varepsilon_{0} h^{2}} \sqrt{\left.\frac{3}{8 \pi G \rho_{\Lambda}}\right]}\right.\right.}}
$$

In the above equation $m_{e}$ is the rest mass of the electron. Recall that Equation (17) and (18) are based purely on classical electrodynamics. However, these results show us that we could have arrived at the same equations if we had made the following two assumptions at the beginning of the analysis. (a) The minimum oscillating charge that can radiate in an antenna is the elementary charge and (b) The minimum energy associated with a single burst of radiation has to be larger than or equal to the energy of a photon. Of course here we are departing from classical electrodynamics because the quantization of the charge or the concept of a photon is not part of the classical electrodynamics. With these two reasonable assumptions, we end up with the following relationship between the elementary charge and the density of the vacuum energy.

$$
e=\sqrt{\frac{4 \varepsilon_{0} h c}{\pi\left\{+\ln \left[\frac{4 \pi c^{2}}{a_{0}} \sqrt{\left.\left.\frac{3}{8 \pi G \rho_{\Lambda}}\right]\right\}}\right.\right.}}
$$

Note that Equation (17) is identical to Equation (19) except that $q_{0}$ is replaced by $e$. Equation (19) can be written as

$$
\rho_{\Lambda}=\frac{3}{8 \pi G\left\{\frac{a_{0}}{4 \pi c^{2}} \exp \left[\frac{4 \varepsilon_{0} h c}{e^{2} \pi}-\gamma\right]\right\}^{2}}
$$

Note that the basis of the derivation of Equations (17) and (19) is different. Equation (17), which is based purely on classical electrodynamics, shows that the smallest oscillating charge that can generate a burst of energy equal to that of a single photon by an antenna stretched to its limiting dimensions is on the order of the elementary charge. On the other hand, Equation (19) results when we assume that the smallest oscillating charge possible is the elementary charge and the smallest energy that could be produced by a single burst of radiation by an antenna stretched to its limiting dimensions is equal to the energy of a photon.

One can interpret Equation (20) as an alternative derivation of the vacuum energy density of the universe. In fact, this is the first time that a connection between the vacuum energy density and the other atomic constants, including the elementary charge, is implied. If we insert the values of the known parameters into Equation (20), we will obtain $\rho_{\Lambda}=4.3 \times 10^{-10} \mathrm{~J} / \mathrm{m}^{3}$. This is slightly less but a good estimation of the vacuum energy. Indeed, this is the value we have to substitute in Equation (17) to make $q_{0}$ exactly equal to the elementary charge. It 
is important to point out that this result is based on the median value of the radiated energy. In order to distinguish between upper and lower limits of the energy, it is necessary to determine the Hubble radius to an accuracy better than about the Bohr radius. Quantum uncertainties as dictated by uncertainty principle would prohibit a measurement of the Hubble radius to such an accuracy. Thus, the use of median value has its merits. However, this point needs further investigations.

The connection between the vacuum energy density and the elementary charge is not surprising given the fact that both the observable value of the elementary charge and the vacuum energy are controlled by vacuum fluctuations [10]. Moreover, if one treats Equation (19) as an exact relationship, one cannot overlook its prediction that the magnitude of the elementary charge decreases with decreasing vacuum energy density and it becomes zero in the absence of vacuum energy. This may be an indication that the vacuum energy is playing a role in maintaining the elementary charge. Unfortunately, these considerations are beyond the scope of this paper. However, Equation (20) is derived based on several assumptions and more analysis on the derivation of this equation is warranted.

\section{Discussion}

As mentioned in the introduction, a study similar to the one reported here was conducted previously in Paper 1 . There are several differences both in the methodology and the results obtained between that study and the present one. First, in Paper 1 it was observed that the energy dissipation over a given period of time reaches more or less a constant value with increasing $L / \lambda$. However, the results presented above show that instead of reaching a constant value the energy increases slowly (i.e. logarithmically) with increasing $L / \lambda$. This slow increase was not captured in that paper due to rounding off errors caused by the numerical integration. Second, in Paper 1 the energy is calculated over a single period rather than over half a period as was done in the present study. We believe that the energy dissipation over half a period is more appropriate than over a full period because the energy is dissipated in bursts of half period durations. Third, in Paper 1 the radiation generated by a dipole antenna fed by two currents at the center was investigated. Here, the radiating system is reduced to its basic element by placing the antenna over a perfectly conducting ground plane and exciting it by a single current. Notwithstanding all these differences the oscillating charge corresponding to a single photon of energy estimated in Paper 1 does not differ more than a factor of two, approximately, and thus the conclusions made in that paper are confirmed in the present study.

In a previous publication related to a similar topic but with transient currents (i.e. [6]) the radius of the universe was assumed to be equal to the radius of the observable universe which indeed depends on the cosmological time. This made the extracted results time dependent even though the time dependence was weak because the cosmological time appeared inside a logarithmic term. Since the 
results obtained in [6] are order of magnitude estimations, due to this weak time dependence they did not change significantly when the cosmological time is changed over the history of the universe except for the times very close to the beginning of the universe. However, as mentioned earlier, a more correct physical parameter to be used in these analyses is the ultimate Hubble radius (i.e. the maximum radius of the universe) which is a constant and directly coupled to the microscopical physical parameters of the fabric of the universe.

The results presented in the previous section shows that under conditions where the dissipated energy by the antenna for a given charge is a maximum (i.e. $L / \lambda$ reaches its upper limit), at least one elementary charge has to be associated with the oscillating current so that the energy dissipated over half a period to be larger than or equal to that of a photon. However, we have only considered the median value of the energy corresponding to a given value $L / \lambda$ even though the number of photons (or the equivalent energy units) associated with a given charge oscillates between two limits in the vicinity of any given $L / \lambda$. Note that the energy oscillates between upper and lower bounds when the ratio $L / \lambda$ changes by 0.5 . In the vicinity of $L / \lambda \sim 10^{36}$ (corresponding to $2 R_{\infty} / a_{0}$ ) the relative change necessary in this ratio to make the radiated energy jump between the two bounds is infinitesimally small and we are not in a position to determine exactly the value of the charge corresponding to this ratio because neither of the parameters $R_{\infty}$ and $a_{0}$ are known to such an accuracy. Under these circumstances the best one can do is to estimate the median value of the energy and the corresponding charge. Figure 3 depicts the charge associated with the oscillating

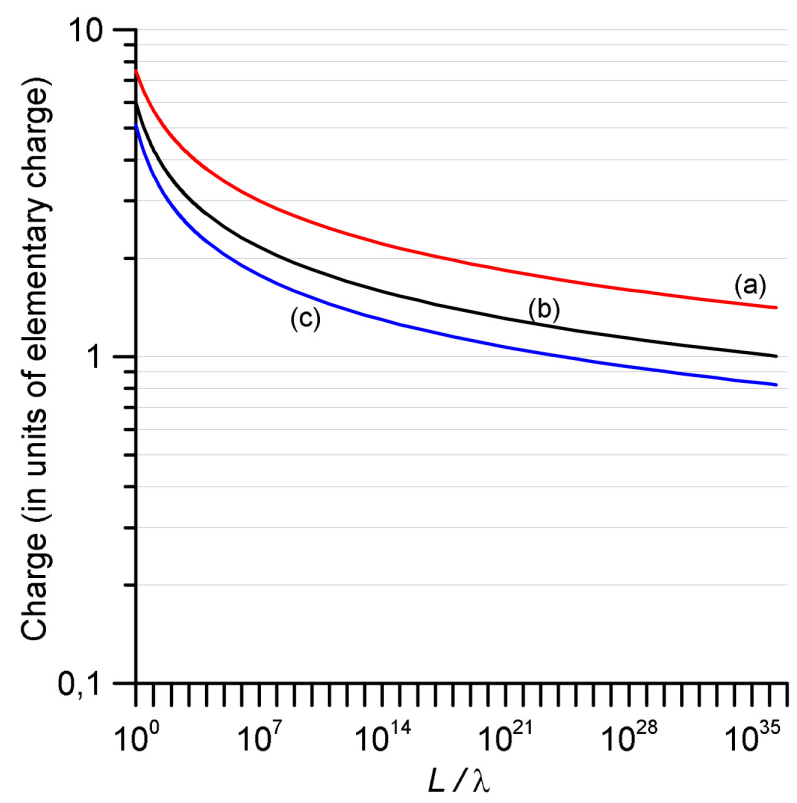

Figure 3. The magnitude of the oscillating charge necessary to generate a one photon of energy over half a period of oscillation as a function of the ratio $L / \lambda$. Results are shown for (a) upper bound, (b) median value and (c) lower bound of Equation (9). 
current for $N=1$ when one estimates it using either the upper bound, lower bound or the median value as given by Equation (9). This shows that in the vicinity of $L / \lambda=R_{\infty} / a_{0}$ even the upper and lower bounds of the charge necessary to generate a single photon within $T / 2$ are still in the order of magnitude of the elementary charge. Thus the results obtained in this paper can be summarized by the order of magnitude relationship $U \geq h v \rightarrow q \geq e$ where $U$ is the energy dissipated over half a period, $q$ is the charge associated with the oscillating current and $e$ is the electronic charge.

Observe that the results presented above are based on absolutely ideal and lossless conditions. In reality, the presence of losses will modify the assumed ideal current distribution and this in turn will give rise to a reduction in the energy radiated. Interestingly, in the presence of losses, a larger charge is necessary to radiate a given amount of energy over a given amount of time than in the ideal case. Thus, the relationship $U \geq h v \rightarrow q \geq e$ still remains valid.

Finally, it is important to stress here that the Equations (17) to (19) given in this paper are based purely on classical electrodynamics. Given the fact that the elementary charge or the concept of photon is not a part of the classical electrodynamics (note that the electron was discovered nearly 30 years after the development of classical electrodynamics), it is remarkable that its predictions lead to the above relationship. Of course, in order to derive an expression for the vacuum energy density we had to utilize both the experimental fact that the electric charge is quantized and the concept of photons from quantum mechanics. However, this semi-classical analysis led to a rather accurate expression for the vacuum energy density as a function of the well-known atomic constants including the elementary charge. However, as mentioned before more investigations are needed on this point.

\section{Conclusions}

The energy radiated by an oscillating current in an antenna occurs in bursts of duration $T / 2$, where $T$ is the period of oscillation. The results obtained here, based purely on classical electrodynamics, can be summarized by the inequality $U \geq h v \rightarrow q_{0} \geq e$ where $U$ is the energy radiated in a single burst of duration $T / 2, h$ is the Planck constant, $v$ is the frequency of oscillation and $q_{0}$ is the magnitude of the oscillating charge associated with the current. The condition $U=h v \rightarrow q_{0}=e$ is obtained when the length of the antenna is equal to the ultimate radius of the universe and the wavelength is equal to the Bohr radius. The inequality obtained here is in general agreement with the one obtained in the previous study.

One novel feature of the analysis is the discovery of an expression in terms of atomic constants including the elementary charge for the vacuum energy density of the universe. This expression predicts the vacuum energy density to be about $4 \times 10^{-10} \mathrm{~J} / \mathrm{m}^{3}$ which is in reasonable agreement with the measured value of about $6 \times 10^{-10} \mathrm{~J} / \mathrm{m}^{3}$. 


\section{Acknowledgements}

Authors appreciate the suggestions, support and encouragement given by Prof. Farhad Rachidi, Prof. Marcos Rubinstein, Prof. Carlo Mazzetti and Prof. Yoshihiro Baba during the development of ideas presented in this paper.

\section{References}

[1] Cooray, V. and Cooray, G. (2016) On the Remarkable Features of the Lower Limits of Charge and the Radiated Energy of Antennas as Predicted by Classical Electrodynamics. Atmosphere, 7, 64. https://doi.org/10.3390/atmos7050064

[2] Cooray, V. and Cooray, G. (2017) Classical Electromagnetic Fields of Moving Charges as a Vehicle to Probe the Connection between the Elementary Charge and Heisenberg's Uncertainty Principle. Natural Science, 9, 219-230.

https://doi.org/10.4236/ns.2017.97022

[3] Balanis, C.A. (1982) Antenna Theory: Analysis and Design. Harper and Row Publishers, New York.

[4] Jackson, J.D. (1975) Classical Electrodynamics. John Wiely \& Sons, New York.

[5] Cooray, V. and Cooray, G. (2016) On the Momentum Transported by the Radiation Field of a Long Transient Dipole and Time Energy Uncertainty Principle. Atmosphere, 7, 151. https://doi.org/10.3390/atmos7110151

[6] Cooray, V. and Cooray, G. (2017) On the Action of the Radiation Field Generated by a Traveling-Wave Element and Its Connection to the Time Energy Uncertainty Principle, Elementary Charge and the Fine Structure Constant. Atmosphere, 8, 46. https://doi.org/10.3390/atmos8030046

[7] Weinberg, S. (2008) Cosmology. Oxford University Press, UK.

[8] Komatsu, E., et al. (2011) Seven-Year Wilkinson Microwave Anisotropy Probe (WMAP) Observations: Cosmological Interpretation. The Astrophysical Journal Supplement Series, 192, 18. https://doi.org/10.1088/0067-0049/192/2/18

[9] Cooray, V., Cooray, G. and Rachidi, F. (2017) Emergence of Friedmann Equation of Cosmology of a Flat Universe from the Time-Energy Uncertainty Principle. Journal of Modern Physics, 8, 1979-1987. https://doi.org/10.4236/jmp.2017.812119

[10] https://en.wikipedia.org/wiki/Quantum_fluctuation 